\title{
Stage II Adrenal Gland Pheochromocytoma and Sympathetic Paraganglioma AJCC v8
}

National Cancer Institute

\section{Source}

National Cancer Institute. Stage II Adrenal Gland Pheochromocytoma and Sympathetic

Paraganglioma A/CC v8. NCI Thesaurus. Code C141130.

Stage II includes: T2, N0, M0. T2: Pheochromocytoma measuring $5 \mathrm{~cm}$ or more in greatest dimension or paragang lioma-sympathetic of any size, with no extra-adrenal invasion. N0: No regional lymph node metastasis. MO: No distant metastasis. (from AJCC 8th Ed.) 